\title{
Trabajo Social Amerindio (TSA)
}

Amerindian Social Work (SWA)

Jorge Alberto Scolar

- Graduado en Servicio Social. Universidade Estadual de Londrina, UEL, 1980.

- Pos grado en Gestión de las Políticas Sociales. Tecnología para la Organización Publica, TOP / Universidad Nacional del Litoral,

Ministério da Saúde. Secretaria Especial de Saúde Indigena, SESAI. Distrito Sanitário Especial Indigena Litoral Sul, DSEI LSUL Dirección postal institucional: Ministénio da 2012

Secretaria Especial de Saúde Indigena, SESAI. Distrit Sanitário Especial Indígena Litoral Sul, DSEI LSUL. E-mail: jorge.scolari@saude.gov.br; joalscolari@gmail.com

\section{Resumen}

El presente artículo sistematiza prácticas de Trabajo Social Indigena desde el ámbito gubernamental que desafian trabajos multidisciplinarios en salud pública intercultural. Desde ellas, se reflexiona sobre la articulación del proyecto ético profesional con equipos multidisciplinares en las unidades de apoyo para tratamiento fuera del hogar, interpretando la integración y el reconocimiento del trabajo socioeducativo con familias indigenas con necesidades sociales especiales. A partir de un enfoque de interdisciplinariedad intersectorial en la gestión de los determinantes sociales, objetivos, teorias y metodos de intervencion social, se presenta un Trabajo Social Amerindio, intercultural, defendiendo el derecho social a la salud asociado con reformas en las politicas públicas indigenas. Finalmente, se abordan las nociones de cohesión social, conciencia colectiva tradicional sanitaria, interdependencia y autonomia, disminuyendo iniquidades, desigualdades y vulnerabilidades.

Palabras clave. Trabajo Social, salud pública indigena, determinantes sociales, enfermedad, cohesión social.

\section{Summary}

This article systematizes indigenous practices done by social workers from government level jobs challenging intercultural multidisciplinary public health. From this point, it analyses the articulation between the ethical professional and multidisciplinary teams in support units for treatment outside home, interpreting integration and recognition of educational work with indigenous families with special social needs. From an interdisciplinary focus in the management of socia determinants, goals, theories and methods of social intervention, this article proposes an Amein indigenous public policy Finally it works the notions of social cohesion, traditional collective health consciousness, interdependence and autonomy reducing inequities, inequalities and vulneabilities.

Key words. Social Work, indigenous public health, social determinants, disease, social cohesion

\section{Introducción}

El Trabajo Social es un trabajador de la salud indígena que interviene prestando un apoyo técnico, social, educativo y asistencial complementario a familias indígenas durante el proceso de trabajo en unidades de atención básica e integral y en diversas otras áreas del sistema de salud pública. ¿Por qué la interdisciplinaridad encuentra fronteras en el $\mathrm{co}_{-}$ tidiano de nuestra historia de vida y profesional? La división social contribuye a la separación entre teoría y práctica, con el fin de controlar, priorizar, monitorear y disciplinar; es en este punto que la práctica interdisciplinaria puede encontrar un lugar para superar la alienación y la exclusión social, a partir de diferentes conocimientos, poniendo en foco la inclusión del trabajo social en las nuevas áreas ocupacionales. El trabajo profesional del trabajador social se gesta en contacto con otros profesionales de diferentes campos; el Trabajo Socia interdisciplinario está directamente relacionado con as acciones de la profesión dentro del marco institucional (tareas, responsabilidades y métodos de trabajo), por lo tanto una práctica interdisciplinaria entre los trabajadores sociales requiere un análisi 
de la sociología de las profesiones, las políticas sociales e institucionales. En las llamadas prácticas de "intervención social" es necesario un enfoque interdisciplinario

Existen diversas definiciones de Trabajo Social, el Conselho Federal de Serviço Social de Brasil, eniende al asistente o trabajador/a social como quien "actúa en el ámbito de las relaciones sociales, junto a individuos, grupos, familias, comunidades y movimientos sociales, desarrollando acciones para fortalecer su autonomía, participación y ciudadanía con el fin de cambiar sus condiciones de vida. Los cial son fundamentales para el trabajo social, con el fin de superar las situaciones de desigualdad social y de violencia, opresión, pobreza, hambre y desempleo" (CFESS 2010). Asimismo, el/la trabajador/a social o asistente social actúa en el ámbito de las relaciones entre los sujetos sociales, y entre ellos y el Estado, desarrollando un conjunto de acciones de carácter socioeducativo que inciden en la reproducción material y social de la vida socil, con individuos, grupos, familias, comunidades y movimientos sociales desde una perspectiva de transformación social. Estas acciones tienen como objetivo fortalecer la autonomía, la participación y el ejercicio de ciudadanía; capacitar movilizar y organizar a los sujetos individual y colectivamente, asegurando el acceso a aienes y servicios sociales. A sienes y servicios socials. A la vez, dichas acciones se orientan a la defensa de los derechos humanos, la salvaguardia de las condiciones socioambientales de existencia, efectivizando los ideales de la democracia y el respeto a la diversidad humana. Así, señala el CFESS (2012), "los principios de defensa de los derechos humanos y la justicia social son fundamentales para el trabajo social, a fin de que dicho rabajo sea desigualdad social y las situaciones de violencia, de opresión, de pobreza, de hambre y desempleo".

En el caso de la Salud Pública Indígena, el objetivo del Trabajo Social es, por una parte, desarrollar la comprensión de los aspectos sociales, económicos y culturales que inciden en el proceso salud - enfermedad y la búsqueda de estrategias pa frente a estas cuestiones, y, por otra, formular es trategias que buscan fortalecer o crear experiencias en servicios de salud que hacen cumplir el derech social a la salud, tomando en cuenta que la labor de los trabajadores sociales en la salud pública tiene como referencia principal el proyecto ético político profesional, necesariamente articulado al proyecto Esto nos da un marco de referencia axiológico para una comprensión mayor de la intervención de los profesionales del Trabajo Social a las demandas necesidades sociales desde la salud indígena.

\section{Metodología de intervención social en}

\section{la interculturalidad}

Para la generación profesional de los años 2000 como un nuevo espacio de ciudadanía social guiada por la Constitución del 88, la atención a la salud indígena constituye una práctica socio política estratégica que confronta al propio sistema público para garantizar el derecho social de acceso a la salud. La actividad de acoger, oír y escuchar, evaluar, articular, orientar y acompañar socialmente familias durante el tratamiento de salud es para algunos, visto como una novedad que se atribuye al proceso de la apertura democrática y de lucha contra la dictadura Sin embargo, para los movimientos sociales históricos que luchan por la causa de las "minorías étnicas y los excluidos" indígenas, negros, gitanos, personas sin hogar, entre otros, eso no tiene nada de nuevo. En consecuencia, la práctica de los trabajadores sociales en materia de salud indígena depende de una referencia clara al proyecto ético-político profesional articulado al de la reforma sanitaria y a la estructura de los servicios sociales en el país donde opera.

En Brasil, desde el año 2000, se opera desde un modelo distrital de atención para la política pública nacional de salud indígena. Esos cambios requieren de parte del Trabajo Social profundizar nuevos conocimientos teóricos, metodológicos, socio jurídicos, culturales, de política indígena e indigenista, de las distintas áreas involucradas en un enfoque inter y multidisciplinar. El Trabajo Social tiene en su amplio campo de acción una cobertura de la cuestión interdisciplinar en varias áreas, lo que hace que la práctica profesional supere a su aprendizaje teórico, y remite al proyecto ético-político de formación profesional, desde la perspectiva del trabajo colectivo. Desde los ámbitos donde se desarrollan los tratamientos, sobre todo fuera de los hogares de las aldeas, suburbios de las ciudades, acompañando las hospitalizaciones en unidades especializadas de complejidad media y alta, el trabajador social desempeña un papel humano intercultural estratégico en la mediación y articulación familiar junto a los equipos multidisciplinares de la salud pública indígena.

Para Faleiros (1981) la abstracción del individuo de la sociedad, y de la sociedad en relación al individuo, ha marcado el problema de la colocación del sujeto y el objeto por el Trabajo Social, ubicado en una visión que aísla las relaciones interindividuales y al propio sujeto del contexto de sus relaciones fundamentales. Define la relación sujeto - objeto solo en una perspectiva histórico-estructural, enfrentando a las connotaciones ideológicas implícitas. El objeto del Trabajo Social se ha establecido acorde a la división tradicional de caso, grupo y comunidad, pero todas esas metodologías aportan una mirada de relaciones, interrelaciones o situaciones que están aisladas del contexto global. Faleiros defiende al sujeto como actor social capaz de, en las relaciones que establece, cambiar su situación. Por situación mismo sujeto. Se mismo sujeto. Se trata de un objeto inmediato, y no de un objeto mediatizado, construido. El circunstancialismo marca profundamente al Trabajo Social. En circunstancias de hambre se da comida; en enfermedad, medicamentos; en situaciones de angustia, un consejo; ante la ignorancia, saber. Se supone que las circunstancias inmediatas determinan al hombre y se olvida que son los hombres los que crean los que cren las circunstancias de hambre, en sus relaciones sociales. En base a esta visión empirista y abstracta del Trabajo Social, que considera al hombre como un producto de estos aspectos separados de la situación, convirtiéndose en objeto de las circunstancias particulares, Faleiros resume las tres perspectivas del trabajo social contemporáneo en: asistencialista, sociológica y tecnocrática.

\section{La génesis del "problema social"} entre los sujetos y actores de la salud indígena

Las condiciones sociales de vida de la mayoría de los pacientes indígenas es de privación de sus necesidades básicas, vulnerabilidad y riesgo de lesiones causadas por las condiciones socioambientales en que viven. Hay una fuerte tendencia a disociar los determinantes sociales de la salud en los tratamientos médicos hospitalarios, moviéndose inconscientemente la culpa de la dolencia al propio paciente. Algunos profesionales se preocupan poco o nada de los aspectos socites, econom poco o na de los aspectos sociales, economicos y como también en la búsqueda de estrategias para hacer frente a estos problemas. Para la Comisión Nacional sobre Determinantes Sociales de la Salud (CDSS), los DSS son los factores sociales, económicos, culturales, étnico-raciales, psicológicos y de comportamiento que influyen en la aparición de problemas de salud y factores de riesgo en la población. La comisión homónima de la Organización
Mundial de la Salud (OMS) tiene una definición más corta, según la cual los DSS son las condiciones sociales en que las personas viven y trabajan. La fragmentación de los derechos sociales llegando de esta forma a la salud, atenta contra los derechos humanos, lo que se transforma en una justicialización continua para corregir las violaciones a los ciudadanos. Independientemente de esto, el modelo distrital de atención del sistema público de salud indígena, continúa siendo estructurado teniendo como focos para la promoción de la salud: la intersectorialidad; las políticas sociales y económicas; a reducción de las iniquidades en el acceso a las acciones; la conciencia acerca del derecho a la salud e importancia de prácticas y hábitos saludables.

En la intersectorialidad, por lo general las políticas son responsabilidad de diferentes sectores, que a menudo operan de forma independiente, lo que obliga al establecimiento a contar con mecanismos que permitan una actuación integrada. Incluye aspectos políticos y técnicos. Para Cunill Grau (2005) en cualquier área donde sea aplicad debemos prestar mucha atención a la institucionalidad política dominante, ya que la misma actú anto como condicionante de la intersectorialidad (positiva o negativamente). En general, esas politicas son responsabilidad de diferentes sectores, que a menudo operan de forma independiente, lo que obliga a establecer mecanismos que permitan una actuación integrada. No son pocos los órganos que están involucrados en la atención de los pueblos indígenas; debemos agruparlos de acuerdo con la naturaleza de la personería jurídica, fines y/o servicios prestados en el área de cobertura, convenios, principales contribuciones e impacto social logrado desde su fundación, nivel de satisfacción de los usuarios-indígenas y liderazgo, misión y visión de la organización social indígena, entre otros. a intersectorialidad, para ser eficaz y eficiente, debe contar con una carta orgánica que contenga las funciones de cada entidad, las relaciones y los flujos de comunicación entre sí, que garanticen la producción conjunta de condiciones favorables para el desarrollo de las comunidades indígenas. Potenciar la capacidad de gestionar con la mayor autonomía posible y sin los vicios del pasado individual y colectivo. El desafío de la relación entre intersectorialidad y capacitación está en produci os bienes para su supervivencia y autosostenibilidad manteniéndose saludables. De estos logro todos extraeremos lecciones.

En esta perspectiva interdisciplinar y multidisciplinar de atención, el Trabajo Social interactúa 
contribuyendo a integrar acciones por medio de la estructuración de redes; articulando bienes y servicios del área socioasistencial con respaldo legal; reduciendo las iniquidades y ampliando el acceso servicios básicos y especiales de salud, reduciendo riesgos y daños a la salud colectiva. Según Margaret Whitehead (1992-2002), las iniquidades en salud entre grupos e individuos son aquellas desigualdes de salud que adios son aquellas desgualdades de salud que además de sistemáticas relevantes son también evitables, injustas e innecesarias, siendo uno de los rasgos más llamativos de la situación sanitaria del Brasil. La mayoría de los paises occidentales usa la ecuación de las iniquidades en la alimentación en relación con el nivel socioe conómico del ingreso familiar, nivel de estudios y ocupación principal para sustentación familiar. En general se reconoce que el ingreso es mediado por la ocupación que desempeñan las personas y que estas a su vez están condicionadas por el nivel educacional. Esta ecuación no sería aplicable a contextos indígenas, en función del diferencial histórico de contacto y de pautas que rigen la lógica de las economías capitalistas de mercado.

\section{Una estrategia intersectorial para} reducir iniquidades biopsicosociales con indígenas

Una correcta identificación de las familias indígenas es esencial para elevar la calidad de vida $y$ gestión de la atención en salud y demás políticas públicas con indígenas; no se justifica una duplicidad institucional de informaciones sociales sino su desburocratización, simplificándola. Así estaremos contribuyendo a reducir iniquidades y desigualdades sociales resguardando los principios bioéticos y des socilur es de los personas por parte de los esionales. Dentro de las nuevas competencias de las unidades integradas en redes, se busca promover la gilidad de referencia y contrareferencia incluyendo acciones de asistencia social y salud pública, as como supervisar la programación y realización de consultas, los exámenes e internación hospitalaria de pacientes indígenas; el registro de las acciones realizadas y ta mantención actualizada de los dosrealizad siens de usu la mi la implantacion y extensión de los registros unificados de las familias indigenas con las adaptaciones necesarias. Una base de datos socioeconómica posibilita el registro e identificación de potenciales beneficiarios de los programas sociales federales contribuyendo a reducir las iniquidades; través de tales registros unificados y actualizados de familias indígenas usuarias se proporciona la localización y características de las mismas, permitiendo al gobierno formular e implementar políticas públicas que promuevan la inclusión social y reducir las iniquidades.

La caracterización socioeconómica y cultural de estas familias permitirá elaborar diagnósticos participativos de sus necesidades sociales prioritarias como familias extensas, no solo en una ciudad sino en todo el territorio del país. Con base en estos datos y necesidades socioeconómicas se contribuirá a mejorar diseños democráticos multiculturales de políticas públicas y programas sociales más focalizados que corrijan iniquidades y desigualdades regionales.

\section{Ambientes sociales de riesgo sanitario}

\section{evitables}

La supervisión estratégica del Gobierno reconoce los territorios indígenas como áreas de mayor preocupación social, en comparación con otros grupos, al ser los daños mayores e irreparables al no haber acceso a las redes de protección social básica, especial para media y alta complejidad en salud. En esta perspectiva, se puede decir que el acceso a los tratamientos en el sistema de salud pública no cambia automáticamente "por un clic cibernético" la condición social ocupacional de sustentabilidad de los pueblos indígenas, pero les devuelve el respeto a la dignidad esencial a través de la autosuperación, y la esperanza de una mejor calidad de vida -en algunos casos, sobrevida- al poder volver a su aldea en estado saludable. Por lo tanto, en la mayoría de los casos, tanto la esperanza de vida como las presiones sociales sufridas son también externas y situacionales, y comienzan en hogares estables o transitorios con bajos o ningún ingreso en su medio social de origen.

\section{Contribución de lo intercultural a la}

afirmación de los derechos sociales indígenas

La visión de la salud, la enfermedad y de los procesos terapéuticos de cura con personas pertenecientes a culturas y comunidades tradicionales indígenas, por diferir de la medicina convencional, exige el abordaje de habilidades, conocimientos complementarios y comprensión de representaciones socioculturales y simbólicas diferentes. La interculturalidad, como uno de los principios generales de las directrices para la política nacional en materia de educación indígena brasilera, se define como todo intercambio positivo y mutuamente enriquecedor entre culturas de diferentes sociedades y de ser una cacuela de la educación a otro diseño dinámico. No pode- mos estar satisfechos solo con "valorizar" o incluso resucitar "contenidos" de culturas "antiguas". Hay que tener en cuenta el constante diálogo entre las culturas, que pueda desentrañar los mecanismos, funciones y dinámicas. Este diálogo supone que la interrelación

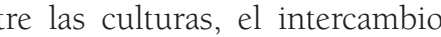
entre ellas y las contribuciones son procesos recíprocos para los cuales todas las sociedades son y han sido sometidos a lo largo de su historia. Estos diferenciales en la percepción de los indígenas frente a equipos multidisciplinarios de salud, revela que requieren, además de personal técnico para la atención primaria que sean personas con preparación, compromiso ético. y sepan tolerar los desafíos que la convivencia presenta en las relaciones interculturales tanto dentro como fuera de los territorios indígenas.

La inseguridad social, la falta de información, la vergüenza, el miedo a lo nuevo y desconocido, más allá de las barreras idiomáticas, son ejemplos de algunos de los indicadores psicosociales que ayudan a comprender la demanda de los diferenciales de calidad en el proceso de acogida, recepción y humanización del cuidado en la atención de la salud con indígenas. Los diferentes grados de adecuación socioambiental y funcional pueden generar ansiedad, inseguridad, miedos, temores e incluso resistencias. Otros grupos que ya han tenido contactos presentan menos resistencia, son más seguros, sin miedos, temores ni ansiedades. Pero el perfeccionamiento del personal de apoyo y del equipo técnico multidisciplinar será crucial en ambas situaciones, lo que facilita la multiplicación de informaciones sobre niveles de satisfacción con base en la atención recibida. A parecer, las familias indígenas que aún no cuentan con el apoyo técnico de profesionales de Trabajo Social en los equipos de salud para orien en la resolución de demandas sociales emergentes, viven un peor proceso de la enfermedad, enfrentan relaciones interpersonales más tensas que afectan el ambiente y la rutina de trabajo, comportamientos con mayores resistencias y sociabilidad, lo que termina atrasando las altas médicas y sociales, o el abandono del tratamiento. Sobre la base de estos problemas es que se desarrolló este trabajo con base en la experiencia práctica de las intervenciones del trabajador social como profesional en equipos multidisciplinarios de salud indígena, con el objetivo de dar una solución técnica adecuada a los problemas sociales, en su mayoría debido a las necesidades humanas básicas insatisfechas (NBI) que se manifiestan durante los tratamientos fuera de los hogares. Estas expresiones sociales constituyen una clara evidencia de cómo los determinantes sociales de la salud son fundamentales para entender la importancia de una perspectiva interdisciplinaria, multidisciplinaria e intersectorial en la salud pública indígena.

\section{La enfermedad como un hecho social}

\section{patológico según Emile Durkheim}

Aún estando previsto en la ley, existe escaso trabajo integrado en los equipos del sistema público de salud indígena. Este hecho impide el desarrollo de una producción y diálogo entre conocimientos populares y científicos escasos y tan útiles y necesarios en el campo de la salud indígena. Al omitir estos aportes de las familias y los "curanderos tradicionales" el sistema sanitario público en general se debilita. Esta es una de las razones por las que se propone crear una alternativa de un Trabajo Social Amerindio (TSA) con una especificidad y una perspectiva interdisciplinar, multidisciplinar e intersectorial en la salud pública indígena. Como hipótesis, a través de estos enfoques es que realmente se contribuye a promover la humanización del cuidado de la salud, el respeto de las especificidades étnico-culturales en terapias ocupacionales con los pacientes y acompañantes, y la articulación con redes de referencia de acciones de la Asistencia Social como política pública con seguimiento y vigilancia necesarias. Contemplar lo social en el proceso de trabajo en salud indígena implica crear oportunidades concretas de desarrollo profesional para actuar en contextos interculturales.

Emile Durkheim entendía a la enfermedad como un hecho social patológico. Para él un hecho normal era aquel que no extrapolaba los límites de los eventos más generales de una determinada sociedad y que reflejaba los valores y comportamientos aceptados por la mayoría de la población. Por ejemplo el matrimonio. El hecho patológico es aquel que está fuera de los límites permitidos por la fuerza social y moral vigente. Los hechos patológicos, como las enfermedades, son considerados temporales y excepcionales. Por ejemplo, un alto índice de criminalidad. Aconsejaba considerar los hechos sociales como cosas, es decir, objetos que están fuera, debiendo ser medidos, observados y comparados independientemente de lo que los individuos involucrados pensasen o declarasen. Esta cosificación en nombre de una ciencia sociológica positivista, pura y neutral excluye el sujeto que experimenta el hecho social patológico. El argumento y los principios positivistas utilizados para romper con las ideas y el sentido común -"especulaciones"- que interpretaban de manera vulgar la realidad social. La práctica 
socioasistencial en la salud indígena muestra en primer lugar, que la cohesión social se despierta es afectada por la enfermedad e invalidez (considerada como un hecho social) de la persona una vez diagnosticada y en tratamiento. Cambios en el área biológica sani po pueden alcanzar directamente o indirectamente a toda la familia indígena en su estructura de parentesco extenso.

La cohesión social como solución ideal para las tensiones y problemas sociales de las ciudades modernas

La cohesión social es concebida como la solución La cohesión social es concebida como la solución
ideal para las tensiones y problemas sociales de las ideal para las tensiones y problemas sociales de las ciudades modernas, como la exclusion de cohesión socia siempre es discutida por las categorías profesionales y la usaremos para profundizar en el debate y ańlisis crítico fundamentadc análisis critico fundannentado que nos permita comprender mejor la transicion de los impactos sociale sufridos por las familias indígenas después del contacto, con todas las contradicciones inherentes a la condición humana y a las relaciones interculturales bajo la influencia del capitalismo. Es propio de la condición humana, y con las familias indígenas no será diferente, el querer ser parte de aloo con lo que cos y distintos. Quieren ser tratadas con igualdad de derechos sociales a las demás, y al mismo tiempo tienen que aprender a valorar la diversidad cultura con la que conviven cuando salen de sus aldeas y territorios. Esta consideración social demuestra la importancia que los desafíos y contradicciones de a modernización y globalización del capitalismo producción capitalista: el cambio y la innovación. Sin embargo, la mayoría de las personas, de acuerdo a sus contextos sociales, aspiran y defienden valore y normas culturales que dan sentido de pertenencia a una especie de bienestar y seguridad social producidos en esos mismos contextos socioculturales en el que nacen, crecen y se reproducen modelos de organización social, económica y política peculiar Por eso hay constantes tensiones entre fuerzas de estabilidad y de transformación socio-económica, como también entre las personas, comunidades y ciudades, que se han visto obligadas a buscar salidas a través de negociaciones que creen nuevas oportunidades y mejoren la calidad de las condiciones de vida, respetando los diversos contextos interculturales y societarios.

Según Durkheim, la intensa división del trabajo social que caracteriza a la sociedad industrial moderna conduce inevitablemente al predominio de la solidaridad orgánica, ya que es en el "día a día", que la interdependencia entre los individuos puede observarse, garantizando la cohesión social, y demostrando el mayor avance social de sociedad industrial en comparación con la sociedad primitiva. El positivismo sociológico de Durkheim trata de diferenciar entre una solidaridad mecánica y otra orgánica. Sus teorías explicativas nos permiten hacer comprender parcialmente la dinámica de la cohesión social en contextos familiares indígenas. En las sociedades precapitalistas, al igual que en algunas de las actuales sociedades tribales indígeindigease, a pesar del tiempo de contacto, los individuos se siguen identificando entre sí a través de la familia, la religión, la tradición y las costumbres. Por lo tanto, desde la matriz familiar permanecen en general independientes y autónomos en relación con la división social del trabajo por mantener su apoyo y supervivencia dentro de las pautas solidarias propias de regímenes agrarios de economía social social familiar comunitaria. La producción de alimentos es cultivada de forma rudimentaria y para el fin solidario del autoconsumo familiar y del clan, de un mismo tronco lingüístico y en territorios de afluencia como su principal fuente de riqueza (Marshall Sahlins, 1984). La conciencia colectiva aquí ejerce todo su poder de coerción sobre los individuos. En la solidaridad orgánica propia de las sociedades capitalistas, por la acelerada división social del trabajo, las personas se tornan interdependientes; esta interdependencia asegura la unión social, en lugar de costumbres, tradiciones y relaciones sociales cercanas. En las sociedades capitalistas, la conciencia colectiva se afloja. Mientras que los individuos son mutuamente dependientes, cada uno se especializa en una actividad y tiende a desarrollar una mayor autonomía personal.

\section{Conclusiones}

La realidad social contemporánea amerindia conlleva las marcas y señales simbólicas de la dominación y alianza ideológica entre las élites monárquicas, religiosas, militares y mercaderes de la "colonización europea". Un proceso de transiciones históricas desde los primeros contactos de esclavitud, seguido del genocidio hasta llegar a una "promoción de la protección social incluyente" conforme a las nuevas fronteras geopolíticas de las actuales naciones del extenso continente americano. La correlación de subordnacion y domina entre tuguesaa de Brasil e hispanoamericana ocurrida a partir del siglo XVI (1500) tendrá un impacto en la infraestructura física, humana, cultural y etnosociopolítica que afectará en su futuro desarrollo y relaciones interculturales.

El diagnóstico sociopolítico actual evidencia expectativas preocupantes ya que pone de relieve el estado de vulnerabilidad biológica y social en que se encuentran los remanecientes, hoy considerados ciudadanos independientes y autónomos en los territorios anexados, o en proceso de regularización como bienes públicos según el ordenamiento socio juŕdico de cada estado nación sudamericano. Las pr. no. Las presiones externas de asimilación cultural sobre la "organización social indígena" evolucionaron con el tiempo evidenciando tensiones cíclicas y ambigüedades dinámicas socioculturales propias interculturales. La transición de "sistemas históricos de necesidades" en la mayoría de las sociedades indígenas contemporáneas en estudio en el sur de Brasil nos demuestran estos diferenciales.

A través de la intervención social intercultural los trabajadores sociales como profesionales de salud en equipos multidisciplinarios, y en otras áreas, impulsan el potencial personal, grupal y comunitario, respetando la afirmación de derechos e identidades. Como tesis, el Trabajo Social Amerindio (TSA), desde una perspectiva interdisciplinaria, multidisciplinar e intersectorial, comenzando desde la salud pública indígena, viene para proponer resignificar las nociones de cohesión social, para que fortalezcan una conciencia colectiva sanitaria tradicional interdependiente, democrática y autónoma. Relación que tiene como objetivo crear más conciencia sobre temas sociales indígenas junto a la sociedad no indígena, desmitificando visiones etnocéntricas gestadas en las relaciones sociopolíticas coloniales y que aún persisten.

La marca de tensión que perdura entre la cohesión y fragmentación de las sociedades amerindias clama por construir colectivamente pactos sociales, a semejanza de movimientos como los "comités para combatir el hambre" de los años 80 en el Braclabil organizada elaboró el primer mapa del hambre, con el trabajo inclusivo de diversos líderes comunitarios en prácticamente todas las sociedades indígenas. Sin un compromiso serio de reordenar una devolución definitiva y pacífica de los territorios con incentivos que promuevan la sostenibilidad con seguridad ambiental y social sanitaria solo veremos crecer la migración de más jóvenes familias indígenas hacia los centos ubanos, que ya es una tendencia en algunas ciudades de mediano tamaño y grandes capitales en la región. La migración transnacional de familias indígenas de Bolivia hacia Sao Paulo es un hecho y fenómeno social asociado a una explotación del trabajo esclavo en pleno siglo XXI.

\section{Referencias bibliograficas}

BRASIL. MEC/SEF/DPEF. (1994) Comitê de Educação Escolar Indígena. Diretrizes para a Política Nacional de Educação Escolar, 2a . Ed. Brasília.

CUNILL, N. (2005) La intersectorialidad en el gobierno y gestión de la politica social. Ponencia presentada en el X Congreso Internacional del CLAD sobre la Reform del Estado y de la Administración Pública, Santiago de Chile.

DURKHEIM, É. (1966) As regras do método sociológico. $4^{a}$ Ed. São Paulo: Editora Nacional.

FALEIROS, V.P. (1981) Metodologia e ideologia do trabalho social. São Paulo: Cortez.

SAHLINS MARSCHALL, D. (1984). Las sociedades tribales. Barcelona. Editorial Labor.

WHITEHEAD, M. (1992) The concepts and principles of equity in heath. International Journal of Health Services

WHITEHEAD, M.; DAHLGREN, G.; GILSON, L. Construcción de una respuesta política a la falta de equidad en la salud: una perspectiva mundial. En: Evans, T.; Whitehead, M.; Diderichsen F; Bhuiva, A.; Wirth, M. (ed.) (2002) Desafio a la falta de Equidad en la Salud: de la ética a la acción. Fundación Rockefeller: Organizay Térnica N.585.

CONSELHO FEDERAL DE SERVICO SOCIAL.

www.cfesss.org.br 